\title{
Convergence Hybrid Network Design for Advanced Multicast and Broadcasting Service based on Mobility Context Awareness Networks: Part II Performance Testing and Analysis CAA Algorithm for Quality of Service
}

\author{
Seungyong Shin and Byungjoo Park ${ }^{*}$ \\ Department of Multimedia Engineering, Hannam University \\ 133 Ojeong-dong, Daeduk-gu, Daejeon, Korea \\ syshin09@hotmail.com,*bjpark@hnu.kr
}

\begin{abstract}
In the case of Mobile IPv6 (MIPv6), which is a typical IP mobility protocol, the host provides the mobility. However, it is considered to be ineffective caused by the increased load causing signaling processing problems. To solve these problems, the Internet Engineering Task Force (IETF) standardized the Proxy Mobile IPv6 (PMIPv6) protocol. PMIPv6 is advantageous in that the mobile terminal does not participate in the signaling procedure related to IP mobility in order to reduce the consumption of radio resources. But in the corresponding mechanism, the multimedia streaming service supported by the PMIPv6 network is always transmitted through the Local Mobility Anchor (LMA) which causes the increase of traffic due to tunnelling and the unnecessary route due to the expansion of the dual tunnel.

Therefore, in this paper, a Context Awareness Algorithm for HFPMPv6 (CAAHFPMIPv6) in order to optimize the multimedia data load distribution according to network situation recognition is proposed. This mechanism is for the cross-layer algorithm design between L2 / L3. For this, the Advanced Mobile Anchor Point (AMAP) concept which is an extension of the hierarchical management method proposed in HMIPv6 (RFC 5380) is introduced. In addition, the Inter Access Point Protocol is used to efficiently manage the network resources through intelligent handover target decision upon handover request, and reverse binding mechanism is used to simplify the handover procedure. Finally, the terminal is assumed to be located between AP overlapping areas of access routers under domain influence.
\end{abstract}

Keywords: IAPP, PMIPv6, FPMIPv6, Reverse Binding Update, Bi-Directional Tunnel

\section{Introduction}

Currently, ubiquitous computing, which enables communication between all objects and smart devices through information infrastructure expansion support, is rapidly evolving. In IPv6, unlike IPv4, fragmentation of a packet to be transmitted is performed only in the terminal host. In the case of Mobile IPv6 (MIPv6), which is a typical IP mobility protocol, the host provides the mobility. However, it is considered to be ineffective caused by the increased load causing signaling processing problems. To solve these problems, the Internet Engineering Task Force (IETF) standardized the Proxy Mobile IPv6 (PMIPv6) protocol.

PMIPv6 is a protocol wherein a Mobile Access Gateway (MAG) supports the mobility on behalf of the mobile terminals, and it is characterized by extending the Access Router

Received (June 26, 2017), Review Result (August 9, 2017), Accepted (August 21, 2017)

* Corresponding Author 
(AR) and Home Agent (HA) functions. Therefore, a MAG having the concept of an AR facilitates the movement of mobile terminals and is managed by the Local Mobility Anchor (LMA) in order to guarantee the mobility of the terminal. In this case, when the handover request is frequent, the network performance may be degraded due to the traffic load concentration problem. Therefore, in order to solve this problem, it is necessary to recognize the network situation information in advance and apply the hierarchical management technique to ensure efficient local mobility. The concept of Hierarchical Fast Handovers for IPv6 (HFPMIPv6) technology, which is based on FMIPv6 (RFC 5568) and HMIPv6 (RFC 5380), is introduced in the PMIPv6 network. This technology concept is similar to the FMIPv6 in a way that it predicts handover signal in L2 layer and tunnel the packets in advance. However, in the case of HMIPv6, the design for IP mobility support is carried out through the same Home Address (HoA) and in PMIPv6, no Duplicate Address Detection (DAD) procedure is required. Therefore, the message format is used only to identify the hierarchical binding procedure.

In this paper, we extend the hierarchical management scheme proposed by HMIPv6 (RFC 5380) in order to reduce Round Trip Time (RTT) through local mobility management and to efficiently transmit the situation information residing in the network to minimize packet loss. Fast Handover for Proxy Mobile IPv6 (FPMIPv6) network and Advanced Mobility Anchor Point (AMAP) device are introduced in order to realize this. In order to more efficiently manage the traffic load concentration problem, the mobility management of the MAGs under the influence of the AMAP domain utilizes the Inter Access Point Protocol (IAPP) capable of collecting preliminary information of the base stations corresponding to the MAG, thus, he handover delay caused by the route optimization procedure is minimized. The rest of this paper is organized as follows: Section 2 describes the existing mobility management techniques and their problems; Section 3 describes the proposed network flow algorithm; in Section 4, performance analysis results of the proposed algorithm are analyzed; and in Section 5, conclusions about the proposed method are derived.

\section{Related Works and Problem}

\subsection{Proxy Mobile IPv6 (PMIPv6)}

PMIPv6 is a protocol that supports mobility within a network domain which is established as RFC5213. It handles the signaling in the network instead of participating in any signaling at the time of handover, so it guarantees the mobility of the mobile station within the restricted network domain. However, since the data load increases due to frequent handover requests and the bottleneck problem are the main causes of packet loss, there is a need for a solution that can effectively disperse such load problems in the future. Figure 1 shows the PMIPv6 handover procedure. 
MN: Mobile Node

NAP: New Access Point

PAP: Previous Access Point

LMA: Local Mobility Anchor
NMAG: New Mobile Access Gateway

PMAG: Previous Mobile Access Gateway

PS/AAA: Policy Store/Authentication Authorization Accounting Server

$\mathrm{CN}$ : Correspondent Node

PMIPv6(Proxy Mobile IPv6)

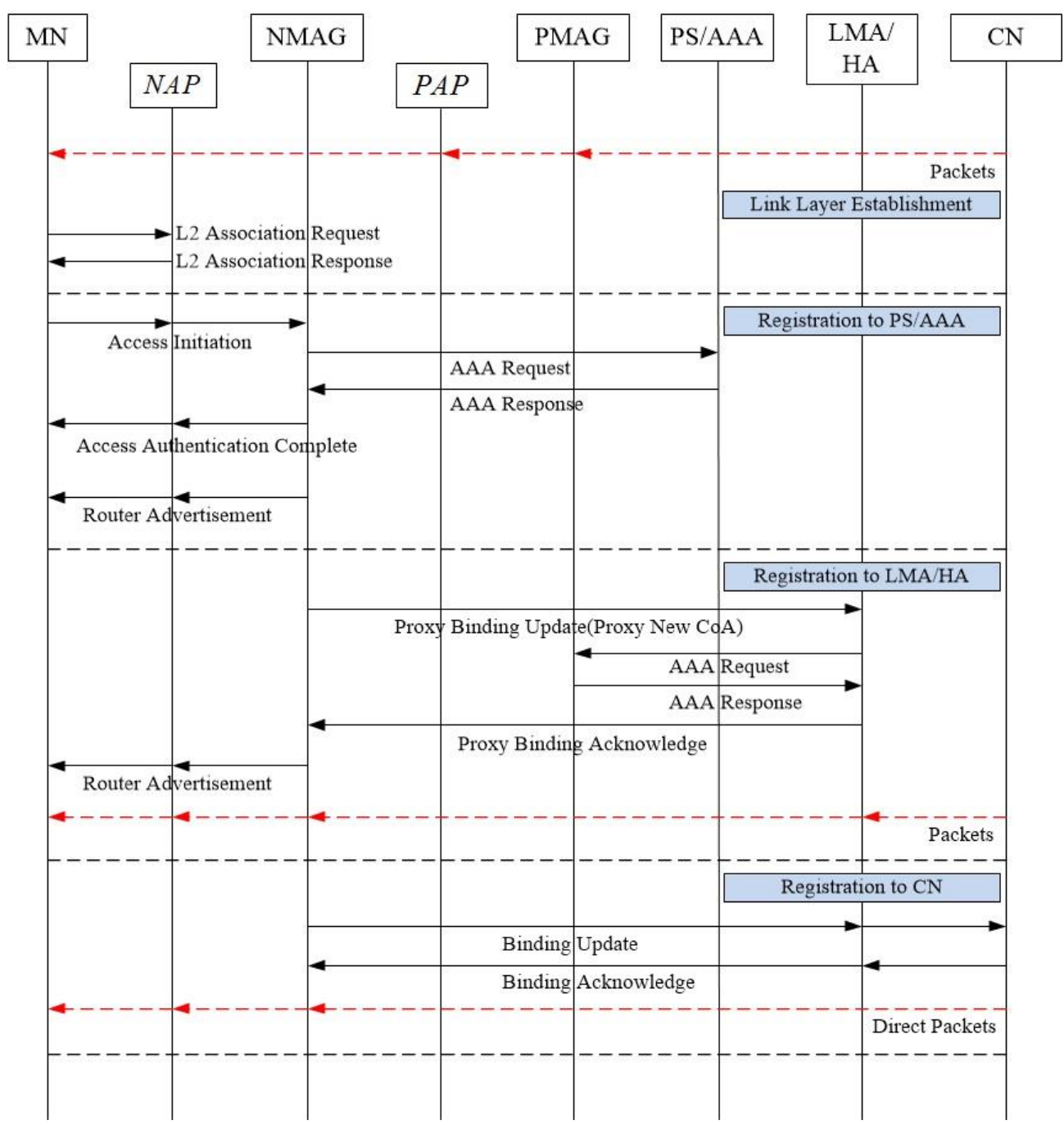

Figure 1. PMIPv6 Handover Procedure

When the PMIPv6 detects the movement from the New Access Point (NAP), it performs the L2 handover procedure to acquire the MN-Identifier (MN-ID). Then, a mutual authentication procedure is performed for ensuring the connectivity between the NMAG and Authentication Authorization Accounting (AAA) server. Proxy Binding Update (PBU) / Proxy Binding Acknowledgment (PBAck) message is exchanged with Local Mobility Anchor (LMA) in order to maintain the Binding Cache Entry (BCE). When the procedure is completed, a bidirectional tunnel is created between the LMA and the MAG, so that all packets transmitted from the $\mathrm{CN}$ after the procedure are transmitted to the MN through the LMA. In order to maintain the routing table between the MAG and the LMA, a Router Advertisement (RA) message under IPSEC protection is periodically transmitted to the MN. PMIPv6 partially solved the problem of signaling load increase due to location registration caused by the handover request which was a problem in the existing host based MIPv6. However, when the data load is increased by performing the part related to providing mobility, there is a possibility of packet loss due to deterioration 
of network performance caused by message transmission procedure for forming a tunnel between the LMA and MAG.

\subsection{Fast Handover for Proxy Mobile IPv6 (FPMIPv6)}

Fast Handover for Proxy Mobile IPv6 (FPMIPv6) is a protocol that helps minimize the packet loss due to the handover delay in the data flow area and reduce the routing overhead in case of handover in transmitting context information residing in the network.

MN: Mobile Node

NAP: New Access Point

PAP: Previous Access Point

LMA: Local Mobility Anchor
NMAG: New Mobile Access Gateway

PMAG: Previous Mobile Access Gateway

PS/AAA: Policy Store/Authentication Authorization Accounting Server $\mathrm{CN}$ : Correspondent Node

FPMIPv6(Fast Handover for Proxy Mobile IPv6)

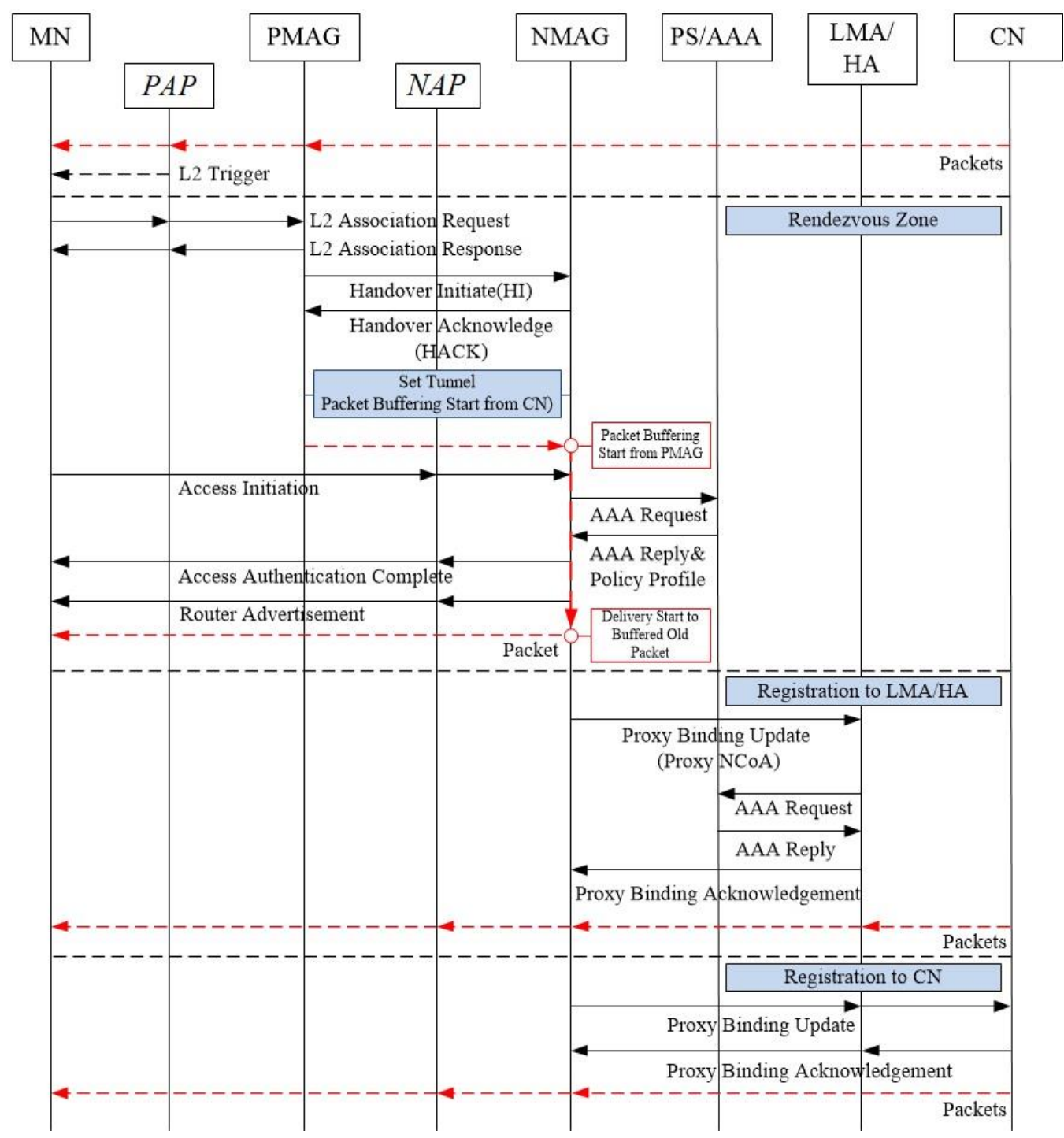

Figure 2. FPMIPv6 Handover Procedure

In addition, the FPMIPv6 extends the function of FMIPv6 in order to guarantee the mobility of the terminal in the PMIPv6 domain, and the terminal does not directly manage mobility due to the nature of the PMIPv6 technology based on the network mobility, FMIPv6 (RFC5568) Router Solicitation for Proxy (RtSolPr), Proxy Router Advertisement 
(PrRtAdv), Fast Binding Update (FBU), and Fask Binding Acknowledgment (FBAck). Therefore, the message format also defines only messages for packet forwarding. Figure 2 shows FPMIPv6 handover procedure in Predictive Mode.

First, the MN detects the handover signal through the L2 Trigger and generates a bidirectional tunnel (HI / HACK) for tunneling the packet. The MN-ID, Home Network Prefix (HNP) and Link Layer Identifier (LL-ID) information are included in the Handover Initiate / Handover Acknowledge (HI / HACK) for this reason. Therefore, the MAG can reduce the handover delay time while performing the binding procedure for the path optimization with the LMA and the CN through the corresponding procedure. However, if the handover request is frequent, the router buffer overflow may occur and it is not so different from MIPv6 in terms of packet loss.

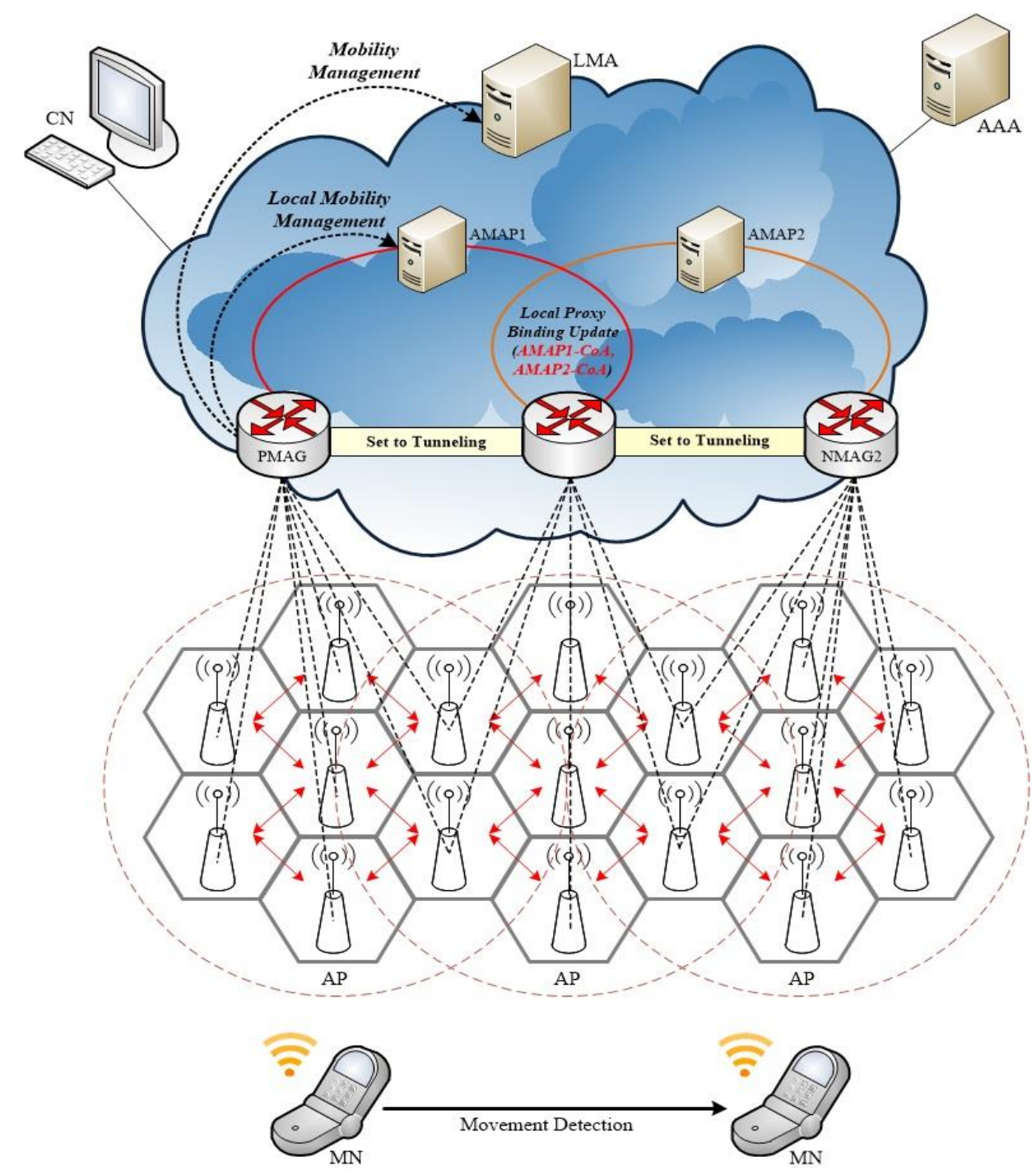

Figure 3. CAA-HFPMIPv6 Conceptual Design Procedure

\section{Routing Optimization using Context-Awareness Algorithm (CAA) for HFPMIPv6 (CAA-HFPMIPv6)}

In this paper, it is assumed that the terminal is located in Access Point (AP) overlap area of access routers under the influence of Advanced Mobile Anchor Point (AMAP) domain in order to demonstrate the efficient local mobility management. Also, in order to construct an organic network by selectively collecting router information, the currently 
connected router regards the gathering of the neighboring network information through the Inter Access Point Protocol (IAPP) as a basic operation. The proposed process procedure are as follows: Figure 3-4 shows the CAA-HFPMIPv6 Conceptual Design Procedure and handover Procedure and Sequence Diagram.
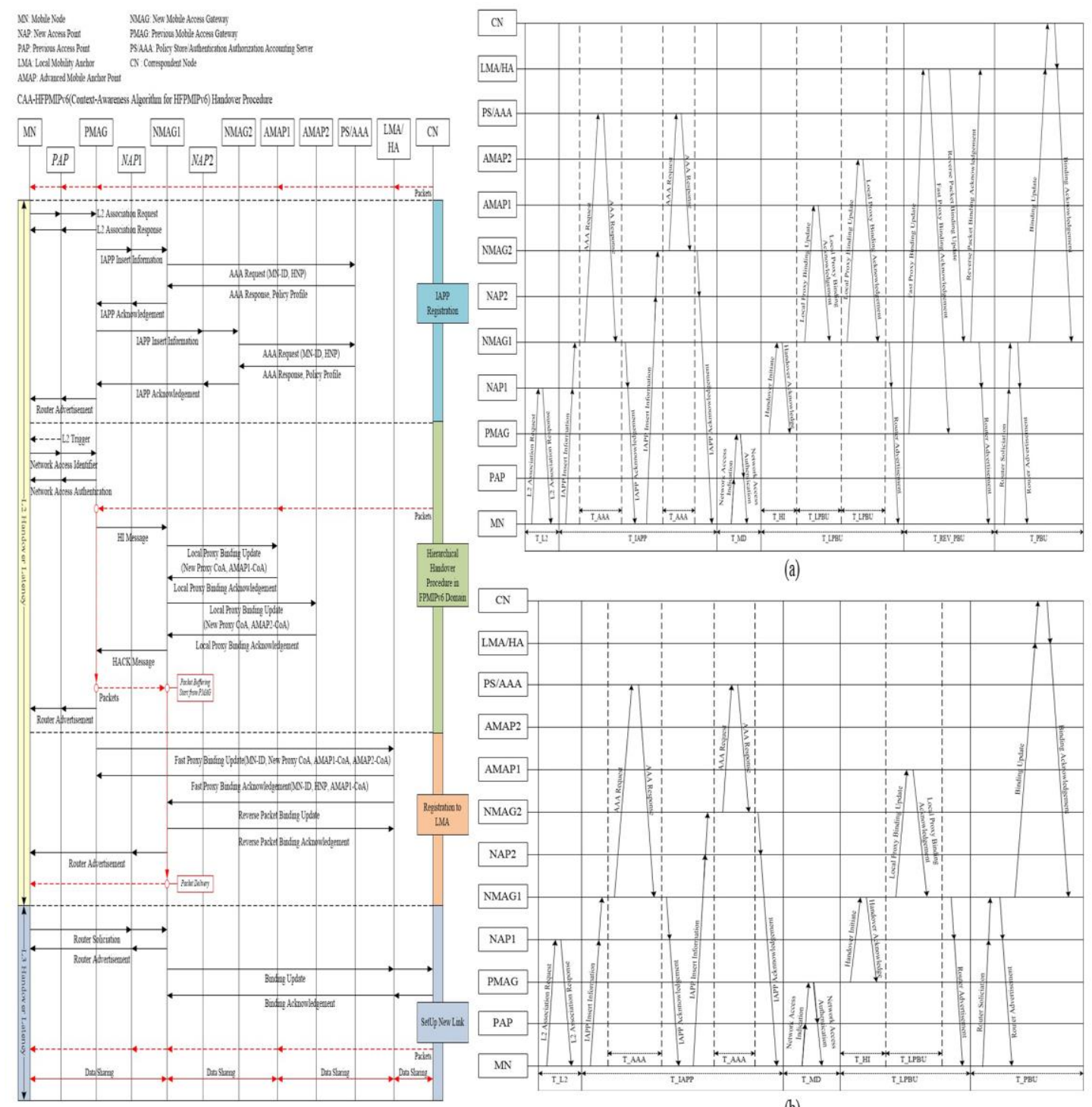

(a)

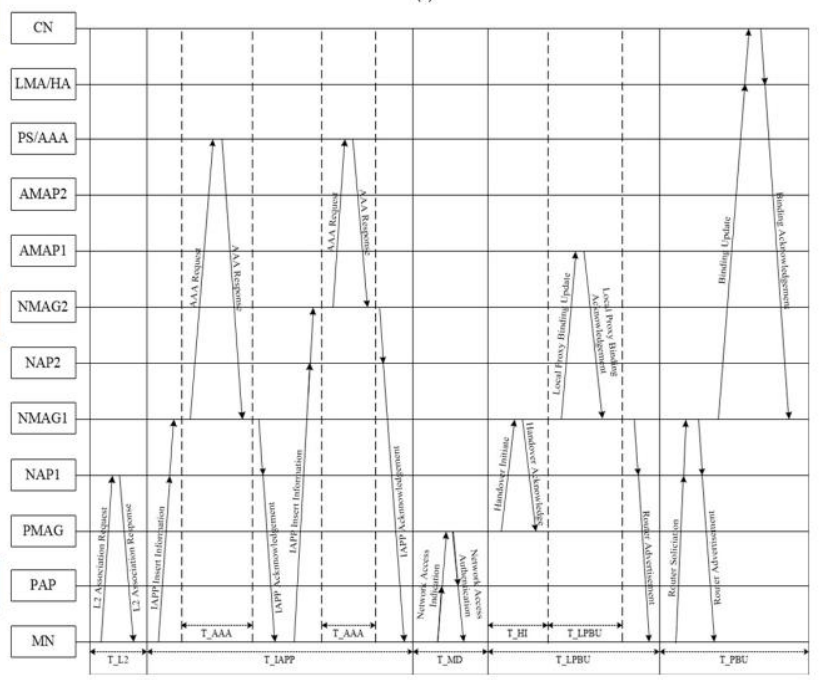

(b)

Figure 4. CAA-HFPMIPv6 Handover Procedure \& Sequence Diagram

1) First, the MN detects the mobility through L2 Trigger signal ( $N_{A P} P_{\text {power }} \geq \operatorname{PAP}_{\text {power }}$ ) and periodic Router Advertisement (RA) messages. At this time, the mobile station identifies the Network Access Identifier (NAI) to and from the currently connected router.

2) Then, the MN sends and receives an Handover Initiate / Handover Acknowledge (HI / HACK) message to form a bidirectional tunnel with the network area NMAG1 to the currently connected router (PMAG). In this paper, we propose a method for selecting a target network by using the information stored in the Policy Store (PS).

3) In the HI / HACK message, the MN-ID, Link Layer Identifier (LL-ID), AMAP1CoA (Care of Address), AMAP2-CoA and Home Network Prefix (HNP) Mobility Option 
are exchanged. In order to create and maintain a binding update list for mandatory items, NMAG1 exchanges Local Proxy Binding Update (LPBU) / Local Proxy Binding Acknowledgment (LPBAck) messages with AMAP1 and AMAP2.

4) The PMAG that creates the bidirectional tunnel through the HI / HACK message delivers the result to the MN through the RA message and at the same time, it transmits the information about the network location to be provided to the Local Mobility Anchor (LMA) Fast Proxy Binding Update (FPBU) / Fast Proxy Binding Acknowledgment (FPBAck) message.

5) The LMA receives the Reverse Proxy Binding Update (RPBU) / Reverse Proxy Binding Acknowledgment (RPBAck) message with the NMAG1 to perform the Reverse Binding Mechanism.

6) After completion of the location registration, the $\mathrm{MN}$ transmits the contents of the corresponding information to the MN through the RA message. In order to perform the Router Discovery and the router route optimization procedure, the MN first transmits a Router Advertisement (RA) message according to the Router Solicitation (RS) and the NMAG1 that receives the Binding Update performs the Binding Update with the LMA / $\mathrm{CN}$ in accordance with the basic procedure specified in PMIPv6 (RFC5213).

Table 1 shows the HI / HACK message format. The message is an extension of the message format specified in FMIPv6 (RFC5568). Based on the information obtained through the IAPP procedure, it determines whether it is within the same domain. If it is determined to be within the same domain, an optional field (H-FLAG) is added in the corresponding message in order to create a temporary tunnel within the message.

Table 1. Handover Initiate/Handover Acknowledge Message Format

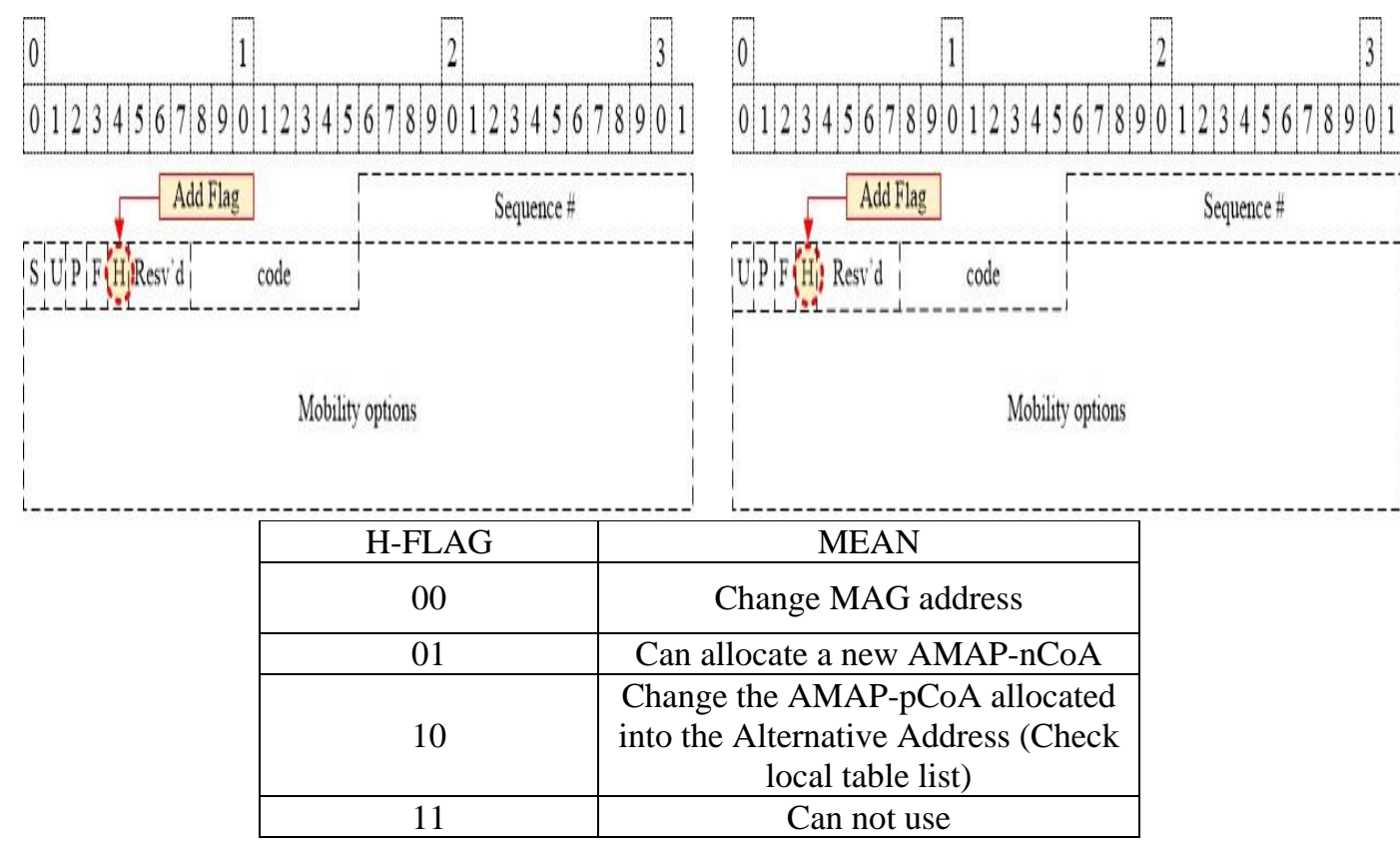

\section{Evaluation \& Comparative Analysis on Performances}

\subsection{The Existing/Suggested Method's Sequence Diagram \& Formula}

The Context Awareness Algorithm for HFPMPv6 (CAA-HFPMIPv6) proposed in this paper assumes that the APs of the routers located in the AMAP domain are located in the wireless overlapping part for managing the local mobility for efficient router selection in 
the same radio access network. In order to verify the performance of the process, the handover delay time between PMIPv6 and CAA-HFPMIPv6 was compared and the packet retransmission rate was considered in order to understand the performance analysis.

Table 2 shows the parameter values for performance analysis. In order to derive the formula, the retransmission rate for each message transmission interval is analyzed, and the CoA generation time of the terminal after receiving Router Advertisement (RA) is set to its maximum of $1000 \mathrm{~ms}$.

$$
\begin{gathered}
T_{\mathrm{CAA} \_ \text {HFPMIP } V}=2 \alpha \beta\left(6 t_{1}+5 t_{2}+2 t_{3}+2 t_{4}+3 t_{5}+t_{6}+\mathrm{t}_{7}\right) \\
T_{\text {CAA_HFPMIPV } 6}=\alpha \beta\left(9 \mathrm{t}_{1}+7 t_{2}+4 t_{3}+2 t_{4}+2 t_{6}\right)
\end{gathered}
$$

Table 2. Performance Analysis Parameters

\begin{tabular}{|c|c|c|}
\hline Symbols & Parameter & Value \\
\hline $\mathrm{t} 1$ & $T_{M N_{-} A P}$ & $10 \mathrm{~ms}$ \\
\hline $\mathrm{t} 2$ & $T_{A P_{-} M A G}$ & $10 \mathrm{~ms}$ \\
\hline $\mathrm{t} 3$ & $T_{M A G_{-} A A A}$ & $10 \mathrm{~ms}$ \\
\hline $\mathrm{t} 4$ & $T_{M A G_{-} M A P}$ & $10 \mathrm{~ms}$ \\
\hline $\mathrm{t} 5$ & $T_{M A G_{-} L M A}$ & $10 \mathrm{~ms}$ \\
\hline $\mathrm{t} 6$ & $T_{M A G_{-} M A G}$ & $10 \mathrm{~ms}$ \\
\hline $\mathrm{t} 7$ & $T_{\text {LMA_CN }_{-}}$ & $10 \mathrm{~ms}$ \\
\hline$\alpha$ & Packet Re-Transmissions Rate & Variable \\
\hline$\beta$ & Packet Loss Rate & Variable \\
\hline
\end{tabular}




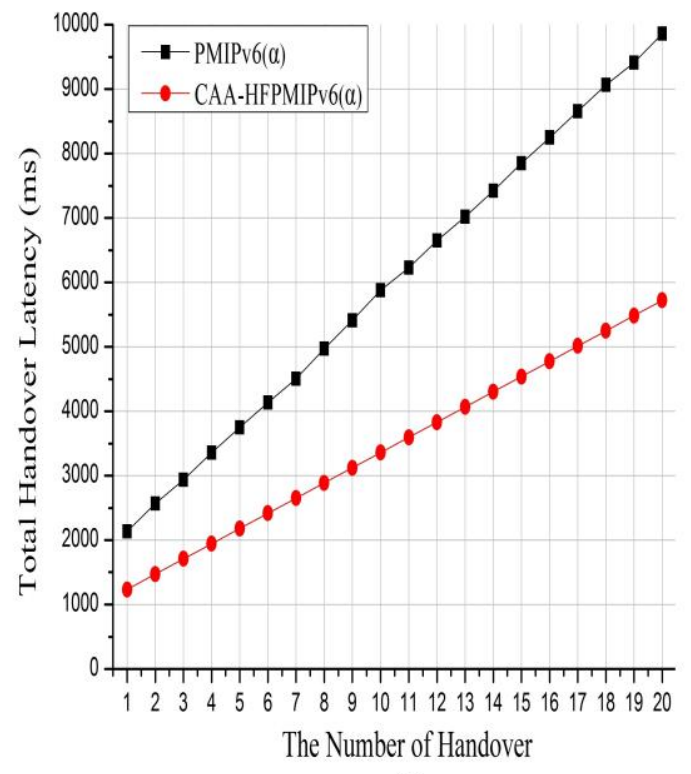

(a)

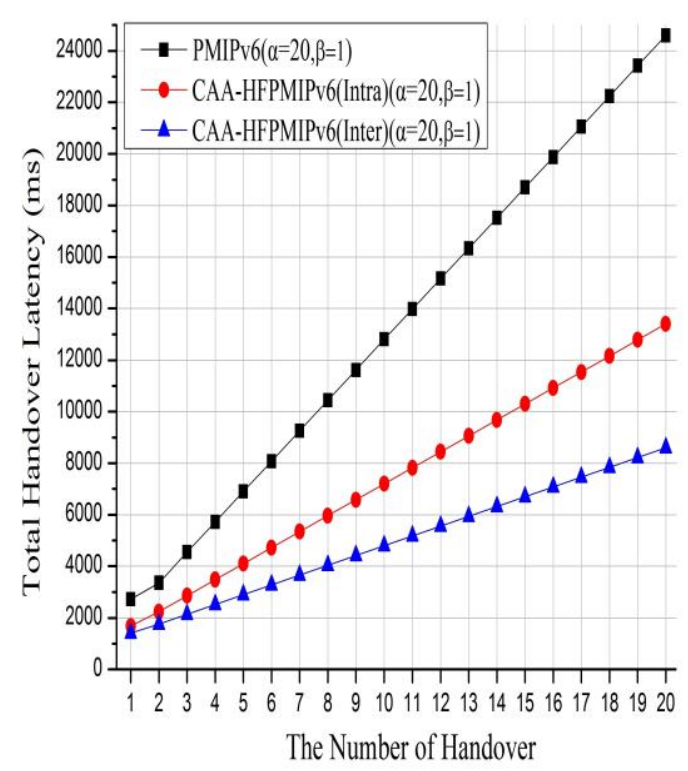

(c)

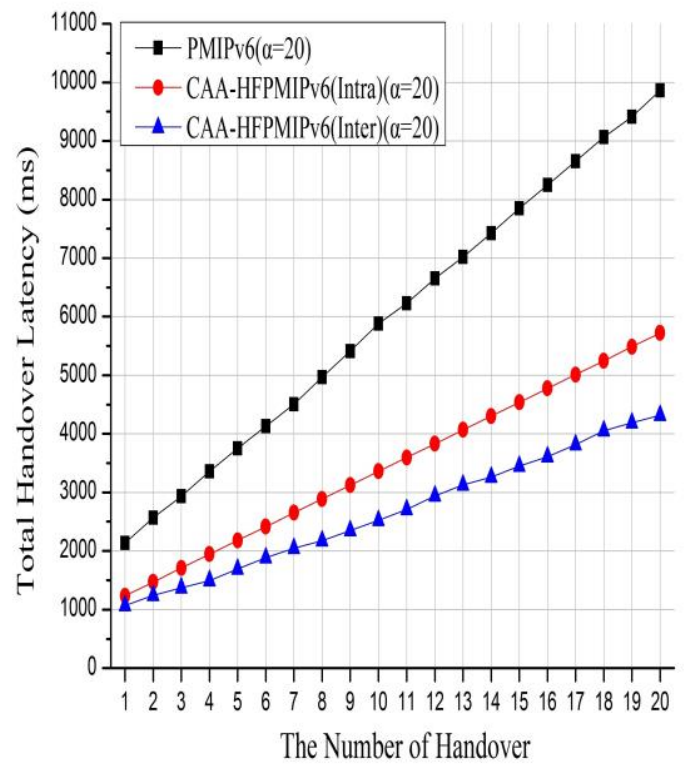

(b)

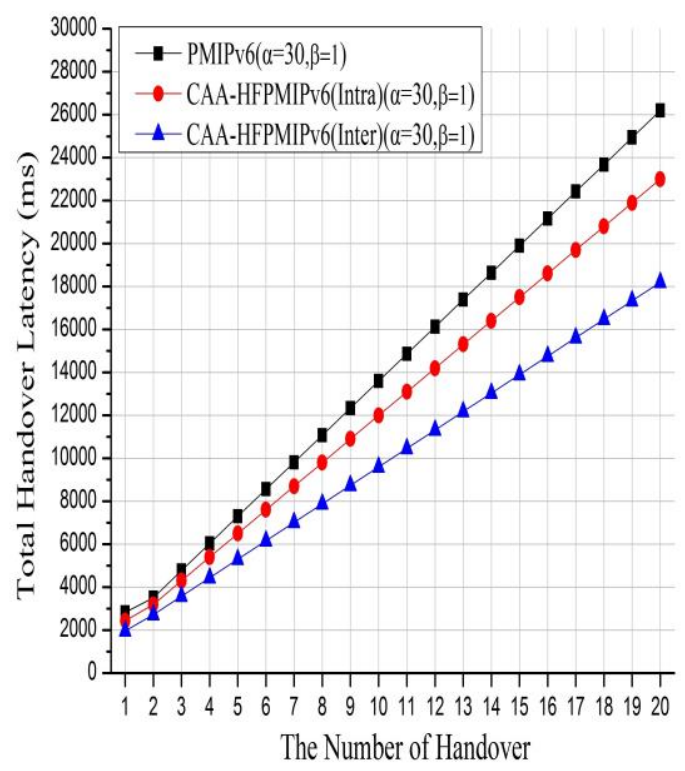

(d)

Figure 5. Performance Test Results Analysis

The $\alpha$ value is a part of the packet retransmission rate that occurs when exchanging packets between routers, and the $\beta$ value is a part of the packet loss rate. Experiments were conducted separately for the part of the packet retransmission rate and the packet loss rate caused thereby. As a result of comparison with the existing PMIPv6, as the frequent handover occurs as shown in Figure 5, the proposed CAA-FHPMIPv6 can be confirmed that the total delay time generated during handover is more efficient than PMIPv6 due to the mobility prediction of the MN. Figure (a) depicts the total delay time due to the increase of the overlapping handover request in the AMAP domain region. The results shows that the pre-shared information between the MAG and AP for traffic 
analysis and efficient prevention of unnecessary signaling channel using radio signal is confirmed to be possible without the existing protocols. Figure (b) depicts the handover latency results based on the increase in the handover requests conducted comparing the differences between the AMAP domain management area. Figure (c), (d) depicts the total handover latency measurement results based on handover requests wherein the packet loss rate increase is set to $20 \%$ and $30 \%$, respectively. The experiments were conducted considering the accuracy in the predictive traffic flow wherein the value of $\beta$ indicates the specific performance that is demonstrated for a change in value of $\alpha$ that is equally applicable to $1 \%$. Thus, the integrated network that is created through the pre-prediction from the analysis of the variety of results during the experiment demonstrated that there is a significant effect on improving the service quality.

\section{Conclusion}

Recently, Internet Engineering Task Force (IETF) has been actively researching and introducing the IPv6 address system, the next generation IP address, to enhance the efficiency of wired / wireless resources. To support the reliable and efficient mobility, PMIPv6 is introduced. However, since the multimedia streaming service supported by the PMIPv6 network is always transmitted through the Local Mobility Anchor (LMA), frequent handover within a limited network area causes complicated signaling, which is a main cause of packet loss. Therefore, in this paper, the CAA-HFPMIPv6 to support advanced fast handover in Fast Proxy Mobile IPv6 (FPMIPv6) is proposed, and the IAPP and AMAP algorithms are introduced to reduce the handover delay time in route optimization, and reverse binding mechanism, respectively. The procedure does not perform the routing path optimization procedure if the mobile station is not in the movement (domain level) within the overlapping AMAP domain to which the mobile station is currently connected, thereby reducing the handover delay time and it is expected that it will provide Quality of Service (QoS) guaranteed service to users when providing real-time multimedia data service over wireless network through the corresponding mechanism in the future.

\section{Acknowledgements}

This research was supported by Basic Science Research Program through the National Research Foundation of Korea (NRF) funded by the Ministry of Science, ICT and future planning (2015R1A2A2A03002851).

\section{References}

[1] T. Narten, E. Nordmark, W. Simpson and H. Soliman, "Neighbor Discovery for IP version 6 (IPv6)", IETF RFC 4861, (2007).

[2] N. Moore and G. Daley, "Fast address configuration strategies for the mobile internet", The Australian Telecommunications, Networks and Applications Conference (ATNAC), (2003).

[3] C. Perkins, D. Johnson and J. Arkko, "Mobility Support in IPv6", IETF RFC 6275, (2011).

[4] H. Soliman, C. Castelluccia, K. ElMalki and L. Bellier, "Hierarchical Mobile IPv6 (HMIPv6) Mobility Management", IETF RFC 5380, (2008).

[5] S. Gundavelli, K. Leung, V. Devarapalli, K. Chowdhury and B. Patil, "Proxy Mobile IPv6", IETF RFC 5213, (2008).

[6] H. Yokota, K, Chowdhury, R. Koodli, B. Patil and F. Xia, "Fast Handovers for Proxy Mobile IPv6", IETF RFC 5949, (2010).

[7] A. Shin Mishra and W. Arbaugh, "An Empirical Analysis of the IEEE 802.11 MAC Layer Handoff Process", ACM SIGCOMM Computer Comm, Rev., vol. 33, no. 2, (2003).

[8] D. Johnson, C. Perkins and J. Arkko, "Mobility Support in IPv6", IETF RFC 3775, (2004).

[9] S. Kent, "IP Encapsulating Security Payload (ESP)", IETF RFC 4303, (2005).

[10] R. Koodli, “Mobile IPv6 Fast Handovers”, IETF RFC 5568, (2009).

[11] B. Park, Y.-H. Han and H. Latchman, "A Study on Optimal Fast Handover Scheme in Fast Handover for Mobile Ipiv6 Networks", LNCS, vol. 4412, (2007). 
[12] K. Kong, W. Lee, Y. Han, M. Shin, and H. You, "Mobility management for All-IP mobile networks: Mobile IPv6 vs Proxy Mobile IPv6”, IEEE Wireless Communications, (2008).

[13] C. Makaya and S. Pierre, "An Analytical Framework for Performance Evaluation of IPv6-Based mobility Management Protocols", Wireless Communications, vol. 7, (2008), pp. 972-983.

[14] S. Park, and Y. Choi, "Performance Analysis of Hierarchical Mobile IPv6 in IP-based Cellular Networks", Proceedings of IEEE Personal, Indoor and Mobile Radio Communications, (2003), pp. 28182822.

[15] J.-Y. Choi, S. Yang and J. Jeong, "On QoS Provisioning Based on User Mobility Patterns for Proxy Mobile IPv6 Networks”, In Computer Science and its Applications, (2015), pp. 983-989. 
International Journal of Future Generation Communication and Networking Vol.10, No.9 (2017) 